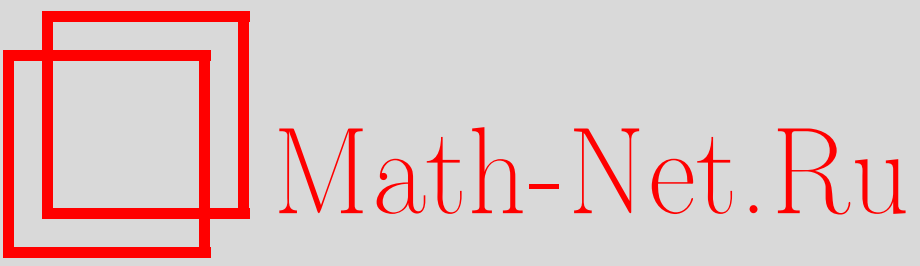

Н. А. Зинченко, О решении аддитивных бинарных задач с полупростыми числами специального вида, Итоги науки и техн. Сер. Соврем. мат. и ее прил. Темат. обз., 2019, том 166, 22-40

DOI: https://doi.org/10.36535/0233-6723-2019-166-22-40

Использование Общероссийского математического портала Math-Net.Ru подразумевает, что вы прочитали и согласны с пользовательским соглашением

http://www.mathnet.ru/rus/agreement

Параметры загрузки:

IP : 54.174 .149 .18

26 апреля 2023 г., 13:23:12 


\title{
О РЕШЕНИИ АДДИТИВНЫХ БИНАРНЫХ ЗАДАЧ С ПОЛУПРОСТЫМИ ЧИСЛАМИ СПЕЦИАЛЬНОГО ВИДА
}

\author{
(c) 2019 г. $\quad$ Н. А. ЗИНЧЕНКО
}

\begin{abstract}
АннотАция. Работа посвящена методам решения бинарных аддитивных задач с полупростыми числами, которые образуют достаточно «редкие» подпоследовательности натурального ряда. На эти числа накладываются дополнительные условия, главное из которых - принадлежность так называемым виноградовским промежуткам. Решаются две задачи, являющиеся аналогами проблемы делителей Титчмарша - выводятся асимптотические формулы для числа решений диофантовых уравнений с полупростыми числами специального вида. Используется метод тригонометрических сумм И. М. Виноградова.
\end{abstract}

Ключевые слова: бинарная аддитивная задача, тригонометрическая сумма, простое число, полупростое число, короткий промежуток.

\section{ON ADDITIVE BINARY PROBLEMS WITH SEMIPRIME NUMBERS OF A SPECIFIC FORM}

\author{
(c) 2019 N. A. ZINCHENKO
}

\begin{abstract}
The paper is devoted to methods of solution of binary additive problems with semiprime numbers, which form sufficiently "rare" subsequences of the natural series. Additional conditions are imposed on these numbers; the main condition is belonging to so-called Vinogradov intervals. We solve two problems that are analogs to the Titchmarsh divisor problem; namely, based on the Vinogradov method of trigonometric sums, we obtain asymptotic formulas for the number of solutions to Diophantine equations with semiprome numbers of a specific form.
\end{abstract}

Keywords and phrases: binary additive problem, trigonometric sum, prime number, semiprime number, short interval.

AMS Subject Classification: 11D75

1. Введение. В аддитивной теории чисел определенное направление посвящено решению задач с простыми числами из так называемых виноградовских (коротких) промежутков:

$$
\left[(2 m)^{c},(2 m+1)^{c}\right), \quad m \in \mathbb{N}, \quad c \in(1,2] .
$$

Заметим, что если обозначить через $\{a\}$ дробную часть числа $a$, то принадлежность простого числа промежутку (1) равносильна условию

$$
\left\{\frac{1}{2} p^{1 / c}\right\}<\frac{1}{2}
$$

Указанное направление возникло в связи с работами И. М. Виноградова [2] и С. А. Гриценко [5]. Аддитивные задачи с простыми числами из промежутков вида (1) рассматривались, например, в $[6,7,13,15]$. Задачи из этих работ были либо тернарными, либо решались по схеме решения тернарной задачи, которую нельзя применить в случае аддитивных бинарных задач с простыми 
числами из виноградовских промежутков. Для решения таких задач требуется аналог теоремы Бомбьери-Виноградова (см. [1,14]), равный ей по силе, который пока не получен. Можно выделить теорему Д. Толева (см. [17], в которой было доказано, что, если выполняются неравенства $0<\lambda<1 / 4,0<\theta<1 / 4-\lambda, A>0$, то

$$
\sum_{k \leqslant x^{\theta}} \max _{y \leqslant x} \max _{\operatorname{gcd}(a, k)=1}\left|\psi_{\lambda}(y ; k, a)-\frac{y^{1-\lambda}}{\varphi(k)(1-\lambda)}\right| \leqslant \frac{x^{1-\lambda}}{\ln ^{A} x}
$$

где

$$
\psi_{\lambda}(y ; k, a)=\sum_{\substack{n \leqslant y \\ n \equiv a \\\{\sqrt{n}\}<1 / n^{\lambda}}} \Lambda(n) .
$$

В этой теореме граница изменения параметра $k$ меньше, чем $x^{1 / 4}$, в то время как в классической теореме Бомбьери-Виноградова она близка к $x^{1 / 2}$. Поэтому применить теорему Толева к решению бинарных аддитивных задач с простыми числами из промежутков вида (1) не удается. В [8] для частного случая эта граница приближена к $x^{1 / 3}$, но и этого недостаточно.

В настоящее время удается решать бинарные аддитивные задачи с полупростыми числами из (1). Это числа вида $p_{1} p_{2}$ или $p_{1} p_{2}^{a}$. Они образуют достаточно редкие последовательности в множестве натуральных чисел. При $a \geqslant 2$ вторая из них близка к последовательности простых чисел. В работе будут рассмотрены две бинарные бинарные аддитивные задачи с этими числами из «виноградовских» промежутков, которые можно считать аналогами проблемы делителей Титчмарша. Задачи решаются методом тригонометрических сумм И. М. Виноградова. Методами, которыми решаются задачи, представленные в работе, можно решать и некоторые другие бинарные задачи с полупростыми числами из «виноградовских» промежутков.

2. Обозначения и вспомогательные леммы. Будем использовать следующие обозначения: $p, p_{1}, p_{2}$ - простые числа;

$$
\begin{aligned}
& \pi(x)=\sum_{p \leqslant x} 1-\text { количество простых чисел, не превосходящих } x ; \\
& \pi(x, a, k)=\sum_{\substack{p \leqslant x \\
p \equiv a(\bmod k)}} 1 ;
\end{aligned}
$$

$\tau(m)$ - количество натуральных делителей числа $m$;

$\mu(m)$ - значение функции Мёбиуса от $m$;

$\varphi(m)$ - значение функции Эйлера от $m$;

$\Lambda(n)$ - значение функции Мангольдта от $m$;

$\operatorname{Li} x=\int_{2}^{x} \frac{d u}{\ln u}$;

$\{x\}$ - дробная часть числа $x$;

$\operatorname{gcd}(a, b)$ - наибольший общий делитель чисел $a$ и $b$;

$[a, b]$ - наименьшее общее кратное чисел $a$ и $b$;

$f(x) \sim g(x)$ означает, что $\lim _{x \rightarrow \infty} \frac{f(x)}{g(x)}=1$;

$A \asymp B$ означает, что существуют такие $c_{1}$ и $c_{1}$, что $c_{1} B \leqslant A \leqslant c_{2} B$.

Для доказательства теоремы используются следующие вспомогательные утверждения.

Лемма 1 (теорема Бруна-Титчмарша, см. [12]). Для натуралънъх чисел а $и$, удовлетворяющих условиям $(a, k)=1 u k \leqslant x$, имеем

$$
\pi(x, a, k)=\sum_{\substack{p \leqslant x, p \equiv a(\bmod k)}} 1<\frac{(2+\eta) x}{\varphi(k) \ln \left(\frac{2 x}{k}\right)},
$$

где $\eta>0$ u $x>x_{0}(\eta)$ 
Лемма 2. Пусть $X \geqslant 2 ;$ тогда справедливо равенство

$$
\sum_{m \leqslant X} \frac{1}{\varphi(m)}=c_{0} \ln X+O(1)
$$

Лемма 3 (см. [4, с. 136]). При $N>2$ и иелом положительном l имеем

$$
\sum_{0<m \leqslant N}(\tau(m))^{l} \ll N(\ln N)^{2 l-1},
$$

где

$$
c_{0}=\sum_{r=1}^{\infty} \frac{\mu^{2}(r)}{r \varphi(r)} .
$$

Лемма 4 (теорема Бомбьери-Виноградова). Для всякого $A>0$ найдется такое $B$, что

$$
\sum_{k \leqslant \frac{\sqrt{x}}{\ln ^{B} x}} \max _{\substack{l \equiv l_{0}(\bmod k) \\(l, k)=1}}\left|\pi(x, l, k)-\frac{\operatorname{Li}(x)}{\varphi(k)}\right|=O\left(\frac{x}{\ln ^{A} x}\right) .
$$

Лемма 5 («лемма о стаканчиках» И. М. Виноградова, см. [3]). Пусть $r-$ иелое положительное число, $\alpha$ и $\beta$-действительные числа, $0<\Delta<0,25, \Delta \leqslant \beta-\alpha \leqslant 1-\Delta$. Тогда существует периодическая функиия $\psi(x)$ с периодом 1, обладающая следующими свойствами:

(1) $\psi(x)=1$ в интервале $\alpha+0,5 \Delta \leqslant x \leqslant \beta-0,5 \Delta$;

(2) $0 \leqslant \psi(x) \leqslant 1$ в интервалах $\alpha-0,5 \Delta \leqslant x \leqslant \alpha+0,5 \Delta$ u $\beta-0,5 \Delta \leqslant x \leqslant \beta+0,5 \Delta$;

(3) $\psi(x)=0$ в интервале $\beta+0,5 \Delta \leqslant x \leqslant 1+\alpha-0,5 \Delta$;

(4) $\psi(x)$ разлагается в рлд Фурье вида

$$
\psi(x)=\beta-\alpha+\sum_{m=1}^{\infty}\left(g_{m} e^{2 \pi i m x}+h_{m} e^{-2 \pi i m x}\right),
$$

где $g_{m}$ и $h_{m}$ зависят только от $m, \alpha, \beta, \Delta$, причем

$$
\begin{gathered}
\left|g_{m}\right| \leqslant \frac{1}{\pi m}, \quad\left|h_{m}\right| \leqslant \frac{1}{\pi m}, \quad\left|g_{m}\right| \leqslant \beta-\alpha, \quad\left|h_{m}\right| \leqslant \beta-\alpha, \\
\left|g_{m}\right|<\frac{1}{\pi m}\left(\frac{r}{\pi m \Delta}\right)^{r}, \quad\left|h_{m}\right|<\frac{1}{\pi m}\left(\frac{r}{\pi m \Delta}\right)^{r} .
\end{gathered}
$$

Лемма 6. Пусть $x-$ большое число, $D \leqslant x^{1-\alpha}$, где

$$
0<\alpha<\frac{1}{2}, \quad(l, D)=1, \quad x_{1}<x, \quad x-x_{1}>x^{1-\alpha / 2} .
$$

Тогда

$$
\sum_{x_{1} \leqslant D m+l \leqslant x}(\tau(D m+l))^{k}=O\left(\frac{x-x_{1}}{D}(\ln x)^{a(k)}\right),
$$

где а $(k)$ - константа, зависящая только от $k$.

K бинарным аддитивным задачам относится известная проблема делителей Титмарша. Так стали называть задачу о получении асимптотической формулы для числа решений уравнения $p-1=x y, p \leqslant n$, которую в 1930 г. поставил и решил Э. Титчмарш (см. [16]) в предположении справедливости расширенной гипотезы Римана. В 1960-х гг. Ю. В. Линник с помощью разработанного им дисперсионного метода вывел асимптотическую формулу для этой задачи безусловно (cм. [11]).

Так как пока бинарные аддитивные задачи с простыми числами из коротких промежутков не поддаются решению, то интерес представляет получение аналогов проблемы делителей Титчмарша с полупростыми числами из (1). 
3. Аналог проблемы Титчмарша для полупростых чисел вида $p_{1} p_{2}$ из коротких промежутков. Будем рассматривать уравнение

$$
p_{1} p_{2}-x y=1,
$$

где $p_{1} p_{2} \leqslant n$. Оно решается в переменных $x, y, p_{1}$ и $p_{2}$, где $x$ и $y$-числа натуральные. Простые числа $p_{1}$ и $p_{2}$ удовлетворяют дополнительным условиям $p_{1}>\exp (\sqrt{\ln n})$ и $p_{2}>\exp (\sqrt{\ln n})$.

Обозначим через $T(n)$ число решений уравнения (2). Очевидно,

$$
T(n)=\sum_{\substack{p_{1} p_{2} \leqslant n \\ p_{1}>\exp (\sqrt{\ln n}) \\ p_{2}>\exp (\sqrt{\ln n})}} \tau\left(p_{1} p_{2}-1\right) .
$$

Через $T_{1}(n)$ обозначим число решений уравнения $(2)$ с полупростыми числами $p_{1} p_{2}$ из промежутков (1). Очевидно,

$$
T_{1}(n)=\sum_{\substack{p_{1} p_{2} \leqslant n \\ p_{1}>\exp (\sqrt{\ln n}) \\ p_{2}>\exp (\sqrt{\ln n}) \\\left\{\frac{1}{2}\left(p_{1} p_{2}\right)^{1 / c}\right\}<\frac{1}{2}}} \tau\left(p_{1} p_{2}-1\right)
$$

Теорема 1. Если с-произвольное число из полуинтервала $(1,2], T(n)$ и $T_{1}(n)$ определень соотношениями (3) и (4) соответственно, то выполняется равенство

$$
T_{1}(n)=\frac{1}{2} T(n)+O(n \ln \ln \ln n),
$$

где

$$
T(n) \sim c_{0} n \ln \ln n, \quad c_{0}=\sum_{r=1}^{\infty} \frac{\mu^{2}(r)}{r \varphi(r)} .
$$

Доказательство теоремы 1 разбивается на несколько этапов.

1. Сначала рассмотрим сумму $T_{1}(n)$ и оценим ошибку, которая возникнет, если ограничить промежуток изменения переменной $x$ в уравнении (2). Для этого преобразуем данную сумму с помощью вспомогательной периодической функции с периодом 1:

$$
\varrho(y)= \begin{cases}1, & \text { если } 0 \leqslant y<1 / 2, \\ 0, & \text { если } 1 / 2 \leqslant y<1 .\end{cases}
$$

Имеем:

$$
\begin{aligned}
& T_{1}(n)=\sum_{\substack{p_{1} p_{2} \leqslant n \\
p_{1}>\exp (\sqrt{\ln n}) \\
p_{2}>\exp (\sqrt{\ln n})}} \tau\left(p_{1} p_{2}-1\right) \varrho\left(\frac{1}{2}\left(p_{1} p_{2}\right)^{1 / c}\right)=\sum_{\substack{p_{1} p_{2} \leqslant n, p_{1} p_{2}-x y=1 \\
p_{1}>\exp (\sqrt{\ln n}) \\
p_{2}>\exp (\sqrt{\ln n})}} \varrho\left(\frac{1}{2}\left(p_{1} p_{2}\right)^{1 / c}\right)= \\
& =\sum_{\substack{p_{1} p_{2} \leqslant n, p_{1} p_{2}-x y=1, x \leqslant \sqrt{n} \\
p_{1}>\exp (\sqrt{\ln n}), p_{2}>\exp (\sqrt{\ln n})}} \varrho\left(\frac{1}{2}\left(p_{1} p_{2}\right)^{1 / c}\right)+\sum_{\substack{p_{1} p_{2} \leqslant n, p_{1} p_{2}-x y=1, x>\sqrt{n} \\
p_{1}>\exp (\sqrt{\ln n}), p_{2}>\exp (\sqrt{\ln n})}} \varrho\left(\frac{1}{2}\left(p_{1} p_{2}\right)^{1 / c}\right) .
\end{aligned}
$$

Пусть $P=n^{1 /(\ln \ln n)^{2}} ;$ тогда

$$
T_{1}(n)=2 \sum_{\substack{p_{1} p_{2} \leqslant n, p_{1} p_{2}-x y=1 \\ x \leqslant \sqrt{n} P^{-10} \\ p_{1}>\exp (\sqrt{\ln n}), p_{2}>\exp (\sqrt{\ln n})}} \varrho\left(\frac{1}{2}\left(p_{1} p_{2}\right)^{1 / c}\right)-R_{2}(n)+2 R_{1}(n),
$$


где

$$
\begin{gathered}
R_{1}(n)=\sum_{\substack{p_{1} p_{2}-x y=1, p_{1} p_{2} \leqslant n, \sqrt{n} P^{-10}<x \leqslant \sqrt{n} \\
p_{1}>\exp (\sqrt{\ln n}), p_{2}>\exp (\sqrt{\ln n})}} \varrho\left(\frac{1}{2}\left(p_{1} p_{2}\right)^{1 / c}\right), \\
R_{2}(n)=\sum_{\substack{p_{1} p_{2}-x y=1, x \leqslant \sqrt{n}, y \leqslant \sqrt{n} \\
p_{1}>\exp (\sqrt{\ln n}), p_{2}>\exp (\sqrt{\ln n})}} \varrho\left(\frac{1}{2}\left(p_{1} p_{2}\right)^{1 / c}\right) .
\end{gathered}
$$

При оценке остатков используется лемма 1. Окончательно получаем, что

$$
T_{1}(n)=2 \sum_{\substack{p_{1} p_{2} \leqslant n, p_{1} p_{2}-x y=1, x \leqslant \sqrt{n} P^{-10} \\ p_{1}>\exp (\sqrt{\ln n}), p_{2}>\exp (\sqrt{\ln n})}} \varrho\left(\frac{1}{2}\left(p_{1} p_{2}\right)^{1 / c}\right)+O\left(\frac{n}{\ln \ln n}\right) .
$$

2. Теперь необходимо ограничить промежуток изменения одного из простых чисел $p_{1}$ или $p_{2}$. Преобразуем сумму в правой части равенства (8):

$$
\begin{aligned}
& \sum_{\substack{p_{1} p_{2} \leqslant n, p_{1} p_{2}-x y=1, x \leqslant \sqrt{n} P^{-10} \\
p_{1}>\exp (\sqrt{\ln n}), p_{2}>\exp (\sqrt{\ln n})}} \varrho\left(\frac{1}{2}\left(p_{1} p_{2}\right)^{1 / c}\right)= \\
& =2 \sum_{\substack{p_{1} p_{2} \leqslant n, p_{1} p_{2}-x y=1, x \leqslant \sqrt{n} P^{-10} \\
\exp (\sqrt{\ln n})<p_{1} \leqslant P, p_{2}>\exp (\sqrt{\ln n})}} \varrho\left(\frac{1}{2}\left(p_{1} p_{2}\right)^{1 / c}\right)+2 R_{3}(n)+R_{4}(n),
\end{aligned}
$$

где

$$
\begin{gathered}
R_{3}(n)=\sum_{\substack{p_{1} p_{2} \leqslant n, p_{1} p_{2}-x y=1, x \leqslant \sqrt{n} P^{-10} \\
P<p_{1} \leqslant \sqrt{n}, p_{2}>\exp (\sqrt{\ln n})}} \varrho\left(\frac{1}{2}\left(p_{1} p_{2}\right)^{1 / c}\right), \\
R_{4}(n)=\sum_{\substack{p_{1} p_{2}-x y=1, p_{1} \leqslant \sqrt{n}, p_{2} \leqslant \sqrt{n} \\
p_{1}>\exp (\sqrt{\ln n}), p_{2}>\exp (\sqrt{\ln n}) \\
x \leqslant \sqrt{n} P^{-10}}} \varrho\left(\frac{1}{2}\left(p_{1} p_{2}\right)^{1 / c}\right) .
\end{gathered}
$$

Для оценки остатка $R_{3}(n)$ воспользуемся тем, что $|\varrho(n)| \leqslant 1$, и леммами 1 и 3 . Получим

$$
R_{3}(n) \ll n \ln \ln \ln n .
$$

Оценивая $R_{4}(n)$, разобьем промежуток суммирования по $p_{1}$ :

$$
R_{4}(n) \leqslant \sum_{\substack{p_{1} p_{2}-x y=1 \\ p_{1} \leqslant P, p_{2} \leqslant \sqrt{n} \\ x \leqslant \sqrt{n} P^{-10}}} 1+\sum_{\substack{p_{1} p_{2}-x y=1 \\ P<p_{1} \leqslant \sqrt{n}, p_{2} \leqslant \sqrt{n} \\ x \leqslant \sqrt{n} P^{-10}}} 1=r^{\prime}+r^{\prime \prime}
$$

Вторая из сумм в правой части полученного неравенства оценивается так же, как и для $R_{3}(n)$. Получаем, что

$$
r^{\prime \prime} \ll n \ln \ln \ln n .
$$

Применяя лемму 2, получим

$$
r^{\prime} \ll \sum_{m \leqslant P \sqrt{n}} \tau(m) \ll P \sqrt{n} \ln P \sqrt{n} \ll \frac{n \ln n}{(\ln \ln n)^{2}} \ll n \ln \ln \ln n .
$$

Следовательно, $R_{4}(n) \ll n \ln \ln \ln n$. Подставляя в $(9)$ оценки для $R_{3}(n)$ и $R_{4}(n)$, из (8) получаем

$$
T_{1}(n)=4 \widetilde{T}_{1}(n)+O(n \ln \ln \ln n),
$$


где

$$
\widetilde{T}_{1}(n)=\sum_{\substack{p_{1} p_{2} \leqslant n, p_{1} p_{2}-x y=1, x \leqslant \sqrt{n} P^{-10} \\ \exp (\sqrt{\ln n})<p_{1} \leqslant P, p_{2}>\exp (\sqrt{\ln n})}} \varrho\left(\frac{1}{2}\left(p_{1} p_{2}\right)^{1 / c}\right) .
$$

Рассуждая аналогично, получаем формулу

$$
T(n)=4 \widetilde{T}(n)+O(n \ln \ln \ln n)
$$

где

$$
\widetilde{T}(n)=\sum_{\substack{p_{1} p_{2}-x y=1, \exp (\sqrt{\ln n})<p_{1}<P, p_{2}>\exp (\sqrt{\ln n})}} 1
$$

3. Получим асимптотическую формулу для суммы $\widetilde{T}(n)$. Покажем, что главный член этой формулы по порядку равен $n \ln \ln n$. Заметим, что, если числа $p_{1}$ и $x$ не взаимно просты $\left(\operatorname{gcd}\left(p_{1}, x\right) \neq 1\right)$, то сравнение $p_{1} p_{2} \equiv 1(\bmod x)$ не имеет решений, и слагаемые в сумме $\widetilde{T}(n)$, соответствующие таким $p_{1}$, будут равны нулю. Поэтому далее считаем, что суммирование проходит только по таким $p_{1}$, которые взаимно просты с $x$. Поэтому

$$
\widetilde{T}(n)=\sum_{x \leqslant \sqrt{n} P^{-10}} \sum_{\substack{\exp (\sqrt{\ln n})<p_{1}<P \\ \widetilde{T}(n)}} \sum_{\substack{p_{2} \leqslant \frac{n}{p_{1}} \\ p_{2} \equiv p_{1}^{*}(\bmod x)}} 1,
$$

где $p_{1}^{*}$ - решение сравнения $p_{1} y \equiv 1(\bmod x)$.

Меняя порядок суммирования и используя для внутренней суммы обозначение из леммы 1 , получим, что

$$
\widetilde{T}(n)=\sum_{\exp (\sqrt{\ln n})<p_{1}<P} \sum_{\substack{x \leqslant \sqrt{n} P^{-10} \\ \operatorname{gcd}\left(p_{1}, x\right)=1}} \pi\left(\frac{n}{p_{1}}, p_{1}^{*}, x\right) .
$$

Представим $\widetilde{T}(n)$ в виде

$$
\widetilde{T}(n)=\sum_{\exp (\sqrt{\ln n})<p_{1}<P} \sum_{\substack{x \leqslant \sqrt{n} P^{-10} \\ \operatorname{gcd}\left(x, p_{1}\right)=1}} \frac{\operatorname{Li}\left(\frac{n}{p_{1}}\right)}{\varphi(x)}+r(n),
$$

где

$$
r(n) \leqslant \sum_{\exp (\sqrt{\ln n})<p_{1}<P} \sum_{x \leqslant \sqrt{n} P^{-10}}\left(\pi\left(\frac{n}{p_{1}}, p^{*}, x\right)-\frac{\operatorname{Li}\left(\frac{n}{p_{1}}\right)}{\varphi(x)}\right) .
$$

Используя теорему Бомбьери-Виноградова (лемма 4), получаем:

$$
\sum_{x \leqslant \sqrt{n} P^{-10}}\left|\pi\left(\frac{n}{p_{1}}, p^{*}, x\right)-\frac{\operatorname{Li}\left(\frac{n}{p_{1}}\right)}{\varphi(x)}\right| \ll \frac{n}{p_{1} \ln ^{A} n},
$$

где $A>0$. Поэтому

$$
r(n) \leqslant \frac{n}{\ln ^{A} n} \sum_{\exp (\sqrt{\ln n})<p_{1}<P} \frac{1}{p_{1}} \ll \frac{n \ln \ln n}{\ln ^{A} n} .
$$

После преобразования первого слагаемого в правой части (12) получим

$$
\sum_{\exp (\sqrt{\ln n})<p_{1}<P} \sum_{\substack{x \leqslant \sqrt{n} P^{-10} \\ \operatorname{gcd}\left(x, p_{1}\right)=1}} \frac{\operatorname{Li}\left(\frac{n}{p_{1}}\right)}{\varphi(x)}=s_{1}(n)+O\left(s_{2}(n)\right),
$$


где

$$
\begin{aligned}
& s_{1}(n)=n \sum_{\exp (\sqrt{\ln n})<p_{1}<P} \frac{1}{p_{1} \ln \frac{n}{p_{1}}} \sum_{\substack{x \leqslant \sqrt{n} P^{-10} \\
\operatorname{gcd}\left(x, p_{1}\right)=1}} \frac{1}{\varphi(x)}, \\
& s_{2}(n)=\frac{n}{\ln ^{2} n} \sum_{\exp (\sqrt{\ln n})<p_{1}<P} \frac{1}{p_{1}} \sum_{x \leqslant \sqrt{n} P^{-10}} \frac{1}{\varphi(x)} .
\end{aligned}
$$

Для $s_{1}(n)$ выведем асимптотическую формулу. Сначала вычислим внутреннюю сумму, представив ее в виде разности:

$$
\sum_{\substack{x \leqslant \sqrt{n} P^{-10} \\\left(x, p_{1}\right)=1}} \frac{1}{\varphi(x)}=\sum_{x \leqslant \sqrt{n} P^{-10}} \frac{1}{\varphi(x)}-\sum_{\substack{x \leqslant \sqrt{n} P^{-10} \\ p_{1} \mid x}} \frac{1}{\varphi(x)}
$$

Из леммы 2 следует, что

$$
\sum_{x \leqslant \sqrt{n} P^{-10}} \frac{1}{\varphi(x)}=c_{0} \ln \sqrt{n} P^{-10}+O(1)=\frac{c_{0}}{2} \ln n+O\left(\frac{\ln n}{(\ln \ln n)^{2}}\right),
$$

где

$$
c_{0}=\sum_{r=1}^{\infty} \frac{\mu^{2}(r)}{r \varphi(r)}
$$

Так как для функции Эйлера выполняется неравенство $\varphi(a \cdot b) \geqslant \varphi(a) \cdot \varphi(b)$, то

$$
\sum_{\substack{x \leqslant \sqrt{n} P^{-10} \\ p_{1} \mid x}} \frac{1}{\varphi(x)}=\sum_{x_{1} \leqslant \sqrt{n} / P^{10} p_{1}} \frac{1}{\varphi\left(x_{1} p_{1}\right)} \leqslant \sum_{x_{1} \leqslant \sqrt{n} / P^{10} p_{1}} \frac{1}{\varphi\left(x_{1}\right) \varphi\left(p_{1}\right)} .
$$

С учетом условия $p_{1}>\exp (\sqrt{\ln n})$, формулы $\varphi\left(p_{1}\right)=p_{1}-1$ и леммы 2 , получаем, что

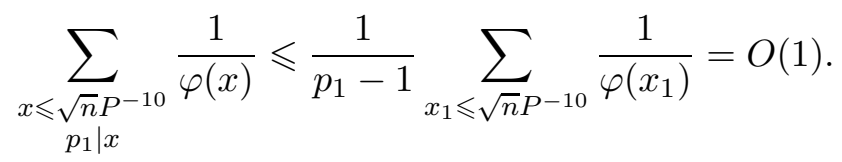

Следовательно,

$$
\sum_{\substack{x \leqslant \leqslant \sqrt{n} P^{-10} \\ \operatorname{gcd}\left(x, p_{1}\right)=1}} \frac{1}{\varphi(x)}=\frac{c_{0}}{2} \ln n+O\left(\frac{\ln n}{(\ln \ln n)^{2}}\right) .
$$

Теперь вычислим сумму

$$
\sum_{\exp (\sqrt{\ln n})<p_{1}<P} \frac{1}{p_{1} \ln \frac{n}{p_{1}}}
$$

Используя преобразование Абеля и формулу

$$
\sum_{p \leqslant n} \frac{1}{p}=C+\ln \ln n+O\left(\frac{1}{\ln n}\right)
$$

которая верна для $n>2$, получаем:

$$
\sum_{\exp (\sqrt{\ln n})<p_{1}<P} \frac{1}{p_{1} \ln \frac{n}{p_{1}}}=\frac{\ln \ln n}{2 \ln n}+O\left(\frac{1}{(\ln n)^{3 / 2}}\right) .
$$

Отсюда, с учетом оценки для внутренней суммы, получаем

$$
s_{1}(n)=\frac{c_{0}}{4} n \ln \ln n+O\left(\frac{n}{\sqrt{\ln n}}\right) .
$$


Для оценки $s_{2}(n)$ применим лемму 2 и формулу для суммы $\sum_{p \leqslant n} \frac{1}{p}$. Получим

$$
s_{2}(n) \ll \frac{n}{\ln ^{2} n} \cdot \ln \ln n \cdot \ln n=\frac{n \ln \ln n}{\ln n} .
$$

Подставляя в (14) асимптотическую формулу для $s_{1}(n)$ и учитывая оценку для $s_{2}(n)$, получаем

$$
\sum_{\exp (\sqrt{\ln n})<p_{1}<P} \sum_{\substack{x \leqslant \sqrt{n} P^{-10} \\ \operatorname{gcd}\left(x, p_{1}\right)=1}} \frac{\operatorname{Li}\left(\frac{n}{p_{1}}\right)}{\varphi(x)}=\frac{c_{0}}{4} n \ln \ln n+O\left(\frac{n}{\sqrt{\ln n}}\right) .
$$

С учетом этой формулы и оценки для $r(n)$ из (12), получаем асимптотическую формулу:

$$
\widetilde{T}(n)=\frac{c_{0}}{4} n \ln \ln n+O\left(\frac{n}{\sqrt{\ln n}}\right), \quad c_{0}=\sum_{r=1}^{\infty} \frac{\mu^{2}(r)}{r \varphi(r)} .
$$

4. Получим асимптотическую формулу для

$$
\widetilde{T}_{1}(n)=\sum_{\substack{p_{1} p_{2} \leqslant n, p_{1} p_{2}-x y=1, x \leqslant \sqrt{n} P^{-10} \\ \exp (\sqrt{\ln n})<p_{1} \leqslant P, p_{2}>\exp (\sqrt{\ln n})}} \varrho\left(\frac{1}{2}\left(p_{1} p_{2}\right)^{1 / c}\right) .
$$

4.1. Воспользуемся леммой 5 и выберем параметры $r, \Delta, \alpha, \beta$ двумя способами. Сначала определим эти параметры так: $r=[\ln n], \Delta=1 / \ln ^{2}(n)$, где $n>\left[e^{2}\right], n \in \mathbb{N}, \alpha=\Delta / 2, \beta=(1-\Delta) / 2$. Легко проверить, что эти числа удовлетворяют условиям леммы 5 . Обозначим функцию $\psi(x)$, существование которой следует из леммы 5 , через $\varrho_{1}(x)$.

Затем, при тех же $r$ и $\Delta$, положим $\alpha=-\Delta / 2, \beta=(1+\Delta) / 2$, а соответствующую функцию обозначим $\varrho_{2}(x)$. Тогда из леммы 5 следует, что $\varrho_{1}(x) \leqslant \chi(x) \leqslant \varrho_{2}(x)$, и

$$
\widetilde{T}_{11}(n) \leqslant \widetilde{T}_{1}(n) \leqslant \widetilde{T}_{12}(n),
$$

где

$$
\widetilde{T}_{1 i}(n)=\sum_{\substack{p_{1} p_{2}-x y=1, p_{1} p_{2} \leqslant n, x \leqslant \sqrt{n} P^{-10} \\ \exp (\sqrt{\ln n})<p_{1}<P, p_{2}>\exp (\sqrt{\ln n})}} \varrho_{i}\left(\frac{1}{2}\left(p_{1} p_{2}\right)^{1 / c}\right), \quad i=1,2 .
$$

Если будут получены асимптотические формулы для $\widetilde{T}_{11}(n)$ и $\widetilde{T}_{12}(n)$ с совпадающими главными членами, то из неравенства (17) следует, что формула с таким же главным членом будет верна и для $\widetilde{T}_{1}(n)$.

Раскладывая функции $\varrho_{1}(x)$ и $\varrho_{2}(x)$ в ряды Фурье, получим:

$$
\begin{aligned}
& \varrho_{1}\left(\frac{1}{2}\left(p_{1} p_{2}\right)^{1 / c}\right)=\frac{1}{2}-\Delta+\sum_{|m| \geqslant 1} g_{1}(m) e^{\pi i m\left(p_{1} p_{2}\right)^{1 / c}}, \\
& \varrho_{2}\left(\frac{1}{2}\left(p_{1} p_{2}\right)^{1 / c}\right)=\frac{1}{2}+\Delta+\sum_{|m| \geqslant 1} g_{2}(m) e^{\pi i m\left(p_{1} p_{2}\right)^{1 / c}},
\end{aligned}
$$

где $\left|g_{i}(m)\right| \leqslant 1 / \pi|m|$ для $i=1,2$. Отсюда и из (11) следует, что

$$
4 \widetilde{T}_{1 i}(n)=\left(\frac{1}{2}+O(\Delta)\right) T(n)+O(\ln \ln \ln n)+4 \sum_{0<|m|<\Delta^{-1} \ln n} g_{i}(m) v_{m}(n),
$$

где

$$
v_{m}(n)=\sum_{\substack{p_{1} p_{2}-x y=1, p_{1} p_{2} \leqslant n, x \leqslant \sqrt{n} P^{-10} \\ \exp (\sqrt{\ln n})<p_{1}<P, p_{2}>\exp (\sqrt{\ln n})}} e^{\pi i m\left(p_{1} p_{2}\right)^{1 / c}}
$$


Следовательно, такая же формула верна и для $4 \widetilde{T}_{1}(n)$ :

$$
4 \widetilde{T}_{1}(n)=\left(\frac{1}{2}+O(\Delta)\right) T(n)+O(\ln \ln \ln n)+4 \sum_{0<|m|<\Delta^{-1} \ln n} g(m) v_{m}(n) .
$$

Подставляя полученное равенство в (10), получим:

$$
T_{1}(n)=\left(\frac{1}{2}+O(\Delta)\right) T(n)+O(\ln \ln \ln n)+4 \sum_{0<|m|<\Delta^{-1} \ln n} g(m) v_{m}(n) .
$$

Следовательно,

$$
T_{1}(n)=\frac{1}{2} c_{0} n \ln \ln n+O(\Delta n \ln \ln n)+O(n \ln \ln \ln n)+4 \sum_{0<|m|<\Delta^{-1} \ln n} g(m) v_{m}(n) .
$$

Теперь требуется оценить последнюю сумму в (19) так, чтобы была получена асимптотическая формула для $T_{1}(n)$.

4.2. Оценка тригонометрической суммы $v_{m}(n)$ представлена в [9]. Рассмотрим вывод этой оценки схематически. Сначала промежуток $(\exp (\sqrt{\ln n}), P]$ суммирования по $p_{1}$ разобьем на промежутки $p_{1} \in\left(P_{1} / 2, P_{1}\right]$, где $P_{1} \leqslant P, P_{1} / 2>e^{\sqrt{\ln n}}>1$, и рассмотрим суммы, соответствующие этим промежуткам:

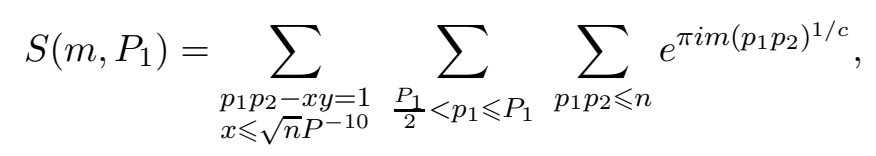

где $P_{1} \in(\exp (\sqrt{\ln n}), P]$. Оценивая эту сумму сверху и применяя неравенство Коши, получим:

$$
\left|S\left(m, P_{1}\right)\right|^{2} \ll \frac{n}{P_{1}} \sum_{\substack{P_{1} / 2<p_{1} \leqslant P_{1} \\ P_{1} / 2<p_{1}^{\prime} \leqslant P_{1}}} \sum_{n_{2} \leqslant \min \left(\frac{n}{p_{1}}, \frac{n}{p_{1}^{\prime}}\right)} \tau^{\prime}\left(p_{1} n_{2}-1\right) \tau^{\prime}\left(p_{1}^{\prime} n_{2}-1\right) e^{\pi i m\left(p_{1}^{1 / c}-\left(p_{1}^{\prime}\right)^{1 / c}\right) n_{2}^{1 / c}},
$$

где

$$
\tau^{\prime}(k)=\sum_{\substack{x y=k \\ x \leqslant \sqrt{n} P^{-10}}} 1
$$

Выделяя диагональные слагаемые $\left(p_{1}=p_{1}^{\prime}\right)$, имеем

$$
\begin{aligned}
& \left|S\left(m, P_{1}\right)\right|^{2} \ll \frac{n}{P_{1}} \sum_{P_{1} / 2<p_{1} \leqslant P_{1}} \sum_{n_{2} \leqslant 2 n / P_{1}} \tau^{2}\left(p_{1} n_{2}-1\right)+
\end{aligned}
$$

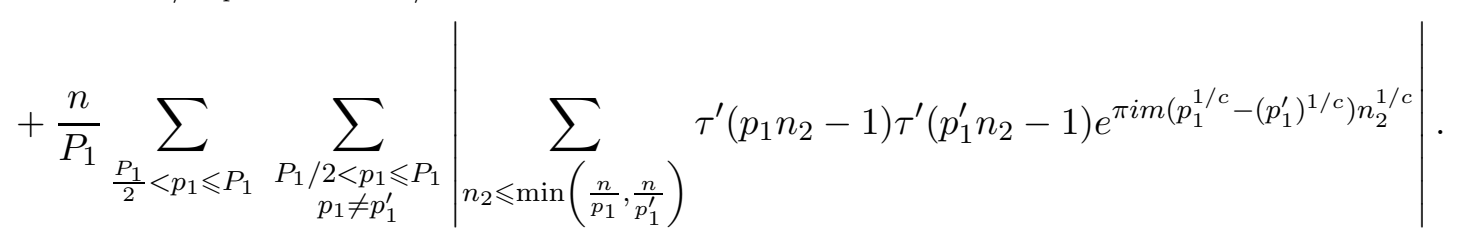

Отсюда, используя для оценки первой суммы лемму 6, получим неравенство

$$
\left|S\left(m, P_{1}\right)\right|^{2} \ll n^{2} \exp \left(-\frac{1}{2} \sqrt{\ln n}\right)+\frac{n}{P_{1}} \sum_{P_{1} / 2<p_{1} \leqslant P_{1}} \sum_{\substack{P_{1} / 2<p_{1}^{\prime} \leqslant P_{1} \\ p_{1} \neq p_{1}^{\prime}}}\left|S\left(m, P_{1}, p_{1}, p_{1}^{\prime}\right)\right|,
$$

где

$$
S\left(m, P_{1}, p_{1}, p_{1}^{\prime}\right)=\sum_{n_{2} \leqslant n_{3}} \tau^{\prime}\left(p_{1} n_{2}-1\right) \tau^{\prime}\left(p_{1}^{\prime} n_{2}-1\right) e^{\pi i m\left(p_{1}^{1 / c}-\left(p_{1}^{\prime}\right)^{1 / c}\right) n_{2}^{1 / c}}, \quad n_{3}=\min \left(\frac{n}{p_{1}}, \frac{n}{p_{1}^{\prime}}\right) .
$$


Очевидно, что

$$
S\left(m, P_{1}, p_{1}, p_{1}^{\prime}\right)=\sum_{x \leqslant \sqrt{n} P^{-10}} \sum_{x_{1} \leqslant \sqrt{n} P^{-10}} \sum_{\begin{array}{c}
n_{2} \leqslant n_{3}, \\
n_{2} \equiv p_{1}^{*}(\bmod x) \\
n_{2} \equiv p_{1}^{\prime *}(\bmod x)
\end{array}} e^{\pi i m\left(p_{1}^{1 / c}-\left(p_{1}^{\prime}\right)^{1 / c}\right) n_{2}^{1 / c}},
$$

где $p_{1} p_{1}^{*} \equiv 1(\bmod x)$ и $p_{1}^{\prime} p_{1}^{* *} \equiv 1(\bmod x)$. Также можно заметить, что, если внутренняя сумма в последнем равенстве не пуста, то $n_{2}$ принадлежит одному из классов вычетов по модулю $\left[x_{1}, x\right]$. Поэтому

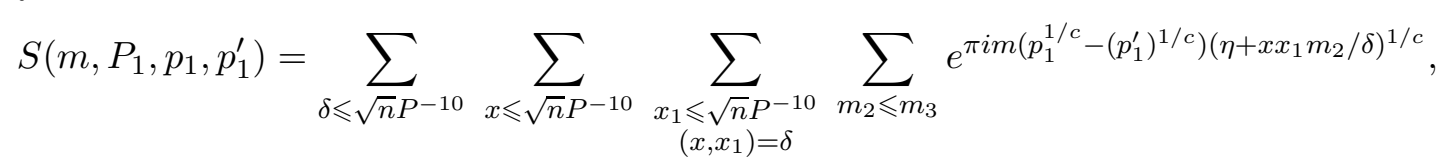

где $m_{3}=\frac{n_{3}-\eta}{\left[x_{1}, x\right]}$ и $0 \leqslant \eta \leqslant\left[x_{1}, x\right]$. Отметим, что сумма по $m_{2}$ «довольно длинная». Например, если $\delta=1$, то $m_{3} \gg P^{19}$.

Обозначим через $\varkappa=m\left(p_{1}^{1 / c}-\left(p_{1}^{\prime}\right)^{1 / c}\right)$ и

тогда

$$
S\left(m_{3}, P_{1}, p_{1}, p_{1}^{\prime}, x, x_{1}, \delta\right)=\sum_{m_{2} \leqslant m_{3}} e^{\pi i \kappa\left(\eta+x x_{1} m_{2} / \delta\right)^{1 / c}}
$$

$$
S\left(m, P_{1}, p_{1}, p_{1}^{\prime}\right)=\sum_{\delta \leqslant \sqrt{n} P^{-10}} \sum_{x \leqslant \sqrt{n} P^{-10}} \sum_{\substack{x_{1} \leqslant \sqrt{n} P^{-10} \\\left(x, x_{1}\right)=\delta}} S\left(m_{3}, P_{1}, p_{1}, p_{1}^{\prime}, x, x_{1}, \delta\right) .
$$

Разобьем промежуток изменения $m_{3}$ по геометрическим прогрессиям и рассмотрим два случая. Если $m_{4} \leqslant m_{3} / P$, то, применяя тривиальную оценку, получим:

откуда следует, что

$$
S\left(m_{3}, P_{1}, p_{1}, p_{1}^{\prime}, x, x_{1}, \delta\right) \ll \frac{m_{3}}{P} \ln n,
$$

$$
S\left(m, P_{1}, p_{1}, p_{1}^{\prime}\right) \ll \frac{n \ln ^{4} n}{P P_{1}} .
$$

Подставляя эту оценку в (20), получим:

$$
\left|S\left(m, P_{1}\right)\right|^{2} \ll n^{2} \exp \left(-\frac{1}{2} \sqrt{\ln n}\right) .
$$

Введем обозначения

$$
\varkappa=m\left(p_{1}^{1 / c}-\left(p_{1}^{\prime}\right)^{1 / c}\right), \quad S\left(m_{3}, P_{1}, p_{1}, p_{1}^{\prime}, x, x_{1}, \delta\right)=\sum_{m_{2} \leqslant m_{3}} e^{\pi i \kappa\left(\eta+x x_{1} m_{2} / \delta\right)^{1 / c}} ;
$$

тогда

$$
S\left(m, P_{1}, p_{1}, p_{1}^{\prime}\right)=\sum_{\delta \leqslant \sqrt{n} P^{-10}} \sum_{x \leqslant \sqrt{n} P^{-10}} \sum_{\substack{x_{1} \leqslant \sqrt{n} P^{-10} \\\left(x, x_{1}\right)=\delta}} S\left(m_{3}, P_{1}, p_{1}, p_{1}^{\prime}, x, x_{1}, \delta\right) .
$$

Разобьем промежуток изменения $m_{3}$ по геометрическим прогрессиям и рассмотрим два случая. Если $m_{4} \leqslant m_{3} / P$, то, применяя тривиальную оценку, получим:

и, следовательно,

$$
S\left(m_{3}, P_{1}, p_{1}, p_{1}^{\prime}, x, x_{1}, \delta\right) \ll \frac{m_{3}}{P} \ln n .
$$

Воспользовавшись этой оценкой, из (20) получим:

$$
S\left(m, P_{1}, p_{1}, p_{1}^{\prime}\right) \ll \frac{n \ln ^{4} n}{P P_{1}} .
$$

$$
\left|S\left(m, P_{1}\right)\right|^{2} \ll n^{2} \exp \left(-\frac{1}{2} \sqrt{\ln n}\right) .
$$


В дальнейшем считаем, что $m_{4}>m_{3} / P$, и рассмотрим сумму

$$
S\left(m_{4}\right)=\sum_{m_{4}<m_{2} \leqslant 2 m_{4}} e^{\pi i \varkappa\left(\eta+x x_{1} m_{2} / \delta\right)^{1 / c}} .
$$

Для случая, когда

$$
0<\frac{|\varkappa|\left(x x_{1} / \delta\right)^{1 / c}}{m_{4}^{1-1 / c}}<\frac{1}{10}, \quad S\left(m_{4}\right) \ll\left(\frac{x x_{1}}{\delta}\right)^{-1 / c} \frac{m_{4}^{1-1 / c}}{|\varkappa|} .
$$

Далее будем рассматривать

$$
\frac{|\varkappa|\left(x x_{1} / \delta\right)^{1 / c}}{m_{4}^{1-1 / c}}>\frac{1}{10} .
$$

Сумма $S\left(m_{4}\right)$ оценивается методом И. М. Виноградова.

Для случая $x x_{1} / \delta>n^{0,99}$ можно применить схему И. М. Виноградова оценки дзетовой суммы (см., например, [10, с. 94-95]), включающую использование «теоремы о среднем». Получаем оценку

$$
S\left(m_{4}\right) \ll m_{4} \exp \left(-\gamma \frac{\ln n}{(\ln \ln n)^{6}}\right) .
$$

Если $x x_{1} / \delta \leqslant n^{0,99}$, то для оценки $S\left(m_{4}\right)$ применим метод ван дер Корпута; в этом случае получаем оценку $S\left(m_{4}\right) \ll \sqrt{m_{4}}$. Окончательно получаем, что

$$
v_{m}(n) \ll n e^{-\frac{1}{4} \sqrt{\ln n}} .
$$

С учетом этой оценки из (18) следует, что

$$
T_{1}(n)=\frac{1}{2} T(n)+O(n \ln \ln \ln n) .
$$

Так как $T(n) \sim c_{0} n \ln \ln n$, то полученная формула является асимптотической. Доказательство теоремы 1 завершено.

4. Аналог проблемы Титчмарша для полупростых чисел вида $p_{1} p_{2}^{a}$ из коротких промежутков. Выведем асимптотическую формулу для числа решений уравнения $p_{1} p_{2}^{a}-x y=1$, где $a \in \mathbb{N}, a \geqslant 2$, числа $p_{1} p_{2}^{a}$ принадлежат промежуткам вида $(1), p_{1} p_{2}^{a} \leqslant n$, и простые числа $p_{1}$, $p_{2}$ удовлетворяют дополнительным условиям.

Теорема 2. Пусть $n \geqslant n_{0}>0, a \geqslant 2$-натуральнье числа, $Q=\exp (\sqrt{\ln n}), A_{1}=\left[1, n Q^{-1}\right]$, $A_{2}=\left[1, Q^{1 / a}\right] u$

$$
G(n)=\sum_{p_{1} \in A_{1}} \sum_{\substack{p_{2} \in A_{2} \\ p_{1} p_{2}^{a}-x y=1}} \sum_{x, y} 1, \quad G_{1}(n)=\sum_{p_{1} \in A_{1}} \sum_{\substack{p_{2} \in A_{2} \\ p_{1} p_{2}^{a}-x y=1 \\\left\{\frac{1}{2}\left(p_{1} p_{2}^{a}\right)^{1 / c}\right\}<\frac{1}{2}}} \sum_{x, y} 1 .
$$

Тогда справедливо равенство

$$
G_{1}(n)=\frac{1}{2} G(n)\left(1+O\left(Q^{-\eta}\right)\right),
$$

где

$$
G(n)=c_{0} \operatorname{Li}\left(\frac{n}{Q}\right) \pi\left(Q^{1 / a}\right) \ln n\left(1+O\left(\frac{1}{\sqrt{\ln n}}\right)\right),
$$

$\eta>0-$ абсолютная постоянная,

$$
c_{0}=\sum_{d=1}^{\infty} \frac{\mu^{2}(d)}{\varphi(d) d}
$$


Доказательство теоремы разбивается на несколько этапов.

1. Получим асимптотическую формулу для $G(n)$. Сначала ограничим промежуток изменения переменной $x$. Получим

$$
G(n)=2 G^{\prime}(n)-G^{\prime \prime}(n)
$$

где

$$
G^{\prime}(n)=\sum_{p_{1} \in A_{1}} \sum_{\substack{p_{2} \in A_{2} \\ p_{1} p_{2}^{a}-x y=1}} \sum_{x \leqslant \sqrt{n}} 1, \operatorname{quad}^{\prime \prime}(n)=\sum_{p_{1} \in A_{1}} \sum_{\substack{p_{2} \in A_{2} \\ p_{1} p_{2}^{a}-x y=1}} \sum_{\substack{x \leqslant \sqrt{n} \\ y \leqslant \sqrt{n}}} 1 .
$$

Оценим сумму $G^{\prime \prime}(n)$, представив ее в виде суммы двух слагаемых $G^{\prime \prime}(n)=G_{1}^{\prime \prime}(n)+G_{2}^{\prime \prime}(n)$, где

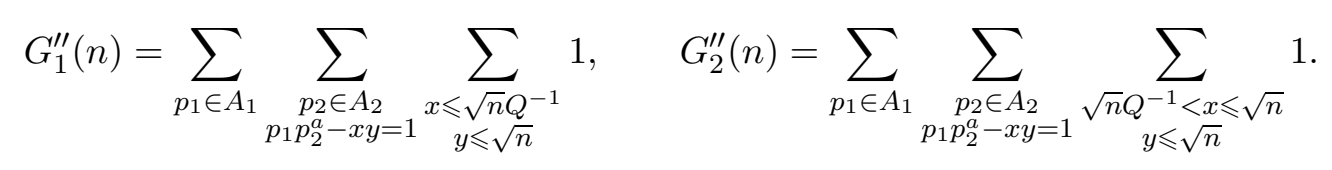

Заметим, что

$$
G_{1}^{\prime \prime}(n) \leqslant \sum_{m \leqslant n Q^{-1}+1} \tau(m-1) t_{1}(m), \quad \text { где } \quad t_{1}(m)=\sum_{\substack{p_{1} p_{2}^{a}=m \\ p_{1} \in A_{1} \\ p_{2} \in A_{2}}} 1 .
$$

Очевидно, что $t_{1}(m)=1$, если $m=p^{a+1}$ или $m=p_{1} p_{2}^{a}$ и $t_{1}(m)=0-$ во всех других случаях. С учетом этого замечания из леммы 3 получаем оценку

$$
G_{1}^{\prime \prime}(n) \leqslant \sum_{m \leqslant n Q^{-1}+1} \tau(m-1) \ll \frac{n \ln n}{Q} .
$$

Оценим $G_{2}^{\prime \prime}(n)$. Так как в случае, когда $p_{2} \mid x$, уравнение $p_{1} p_{2}^{a}-x y=1$ не имеет решений, то

$$
G_{2}^{\prime \prime}(n)=\sum_{\sqrt{n} Q^{-1}<x \leqslant \sqrt{n}} \sum_{\substack{p_{2} \in A_{2} \\ \operatorname{gcd}\left(p_{2}, x\right)=1}} \sum_{\substack{p_{1} \in A_{1} \\ p_{1} \equiv p_{2}^{*}(\bmod x)}} 1=\sum_{\sqrt{n} P Q^{-1}<x \leqslant \sqrt{n}} \sum_{\substack{p_{2} \in A_{2} \\ \operatorname{gcd}\left(p_{2}, x\right)=1}} \pi\left(n Q^{-1}, p_{2}^{*}, x\right),
$$

где $p_{2}^{*}-$ решение сравнения $p_{2}^{a} t \equiv 1(\bmod x)$. Применяя леммы 1 и 2, получаем:

$$
G_{2}^{\prime \prime}(n) \ll \frac{n}{Q \ln n} \sum_{p_{2} \in A_{2}} \sum_{\sqrt{n} Q^{-1}<x \leqslant \sqrt{n}} \frac{1}{\varphi(x)} \ll \frac{n}{Q} \pi\left(Q^{1 / a}\right) \frac{\ln Q}{\ln n} .
$$

Из оценок для $G_{1}^{\prime \prime}(n)$ и $G_{2}^{\prime \prime}(n)$ следует, что

$$
G^{\prime \prime}(n) \ll \frac{n}{Q} \pi\left(Q^{1 / a}\right) \frac{\ln Q}{\ln n} .
$$

Для оценки суммы $G^{\prime}(n)$ представим ее, как и $G^{\prime \prime}(n)$, в виде суммы двух слагаемых:

$$
G^{\prime}(n)=G_{1}^{\prime}(n)+G_{2}^{\prime}(n)=\sum_{p_{1} \in A_{1}} \sum_{\substack{p_{2} \in A_{2} \\ p_{1} p_{2}^{a}-x y=1}} \sum_{x \leqslant \sqrt{n} Q^{-1}} 1+\sum_{p_{1} \in A_{1}} \sum_{\substack{p_{2} \in A_{2} \\ p_{1} p_{2}^{a}-x y=1}} \sum_{\sqrt{n} Q^{-1}<x \leqslant \sqrt{n}} 1 .
$$

Сумма $G_{2}^{\prime}(n)$ оценивается так же, как и $G_{2}^{\prime \prime}(n)$, поэтому

$$
G_{2}^{\prime}(n) \ll \frac{n}{P Q} \pi\left(Q^{1 / a}\right) \frac{\ln Q}{\ln n} .
$$


Рассмотрим сумму $G_{1}^{\prime}(n)$ и получим для нее асимптотическую формулу. Имеем

$$
\begin{aligned}
G_{1}^{\prime}(n)= & \sum_{x \leqslant \sqrt{n} Q^{-1}} \sum_{\substack{p_{2} \in A_{2} \\
\operatorname{gcd}\left(p_{2}, x\right)=1}} \pi\left(\frac{n}{Q}, p_{2}^{*}, x\right)= \\
& =\operatorname{Li}\left(\frac{n}{Q}\right) \sum_{p_{2} \in A_{2}} \sum_{\substack{x \leqslant \sqrt{n} Q^{-1} \\
\operatorname{gcd}\left(p_{2}, x\right)=1}} \frac{1}{\varphi(x)}+O\left(\sum_{p_{2} \in A_{2}} \sum_{\substack{x \leqslant \sqrt{n} Q^{-1} \\
\operatorname{gcd}\left(p_{2}, x\right)=1}}\left|\pi\left(\frac{n}{Q}, p_{2}^{*}, x\right)-\frac{\operatorname{Li}\left(\frac{n}{Q}\right)}{\varphi(x)}\right|\right),
\end{aligned}
$$

где $p_{2}^{*}$ - решение сравнения $p_{2}^{a} t \equiv 1(\bmod x)$. Отсюда, в силу теоремы Бомбьери-Виноградова (лемма 4)

$$
G_{1}^{\prime}(n)=\operatorname{Li}\left(\frac{n}{Q}\right) \sum_{p_{2} \in A_{2}} \sum_{\substack{x \leqslant \sqrt{n} Q^{-1} \\ \operatorname{gcd}\left(p_{2}, x\right)=1}} \frac{1}{\varphi(x)}+O\left(\frac{n \pi\left(Q^{1 / a}\right)}{Q \ln n}\right) .
$$

Для оценки внутренней суммы в первом слагаемом правой части полученного равенства проведем рассуждения, аналогичные тем, что проводились на этапе 3 при доказательстве теоремы 1. Получим

где

$$
\sum_{\substack{x \leqslant \sqrt{n} Q^{-1} \\ \operatorname{gcd}\left(p_{2}, x\right)=1}} \frac{1}{\varphi(x)}=c_{0} \ln \left(\frac{\sqrt{n}}{Q}\right)+O\left(\frac{\ln n}{p_{2}}\right),
$$

$$
c_{0}=\sum_{d=1}^{\infty} \frac{\mu^{2}(d)}{\varphi(d) d} .
$$

Просуммировав обе части полученного равенства по $p_{2} \in A_{2}$, получим

$$
\sum_{p_{2} \in A_{2}} \sum_{\substack{x \leqslant \sqrt{n} Q^{-1} \\ \operatorname{gcd}\left(p_{2}, x\right)=1}} \frac{1}{\varphi(x)}=c_{0} \ln \left(\frac{\sqrt{n}}{Q}\right) \pi\left(Q^{1 / a}\right)+O(\ln n \ln \ln Q) .
$$

Поэтому имеем асимптотическую формулу

$$
G_{1}^{\prime}(n)=c_{0} \operatorname{Li}\left(\frac{n}{Q}\right) \pi\left(Q^{1 / a}\right) \ln \left(\frac{\sqrt{n}}{Q}\right)\left(1+O\left(\frac{\ln Q}{\ln n}\right)\right) .
$$

Отсюда, с учетом оценки для $G_{2}^{\prime \prime}(n)$, получим

$$
G^{\prime}(n)=\frac{c_{0}}{2} \operatorname{Li}\left(\frac{n}{Q}\right) \pi\left(Q^{1 / a}\right) \ln n\left(1+O\left(\frac{\ln Q}{\ln n}\right)\right) .
$$

Окончательно, подставляя (23) и (22) в (21), приходим к асимптотической формуле

$$
G(n)=c_{0} \operatorname{Li}\left(\frac{n}{Q}\right) \pi\left(Q^{1 / a}\right) \ln n\left(1+O\left(\frac{1}{\sqrt{\ln n}}\right)\right) .
$$

2. Рассмотрим сумму

$$
G_{1}(n)=\sum_{p_{1} \in A_{1}} \sum_{\substack{p_{2} \in A_{2} \\ p_{1} p_{2}^{a}-x y=1 \\\left\{\frac{1}{2}\left(p_{1} p_{2}^{a}\right)^{1 / c}\right\}<\frac{1}{2}}} \sum_{x, y} 1
$$

Считаем далее, что $n \geqslant n_{0}>0$, где $n_{0}$ - достаточно большое число. Аналогично тому, как это было сделано при доказательстве теоремы 2, будем использовать вспомогательную периодическую функцию $\chi(x)$, определенную равенством

$$
\chi(x)= \begin{cases}1, & \text { если } 0 \leqslant x<1 / 2 \\ 0, & \text { если } 1 / 2 \leqslant x<1\end{cases}
$$


Очевидно,

$$
G_{1}(n)=\sum_{p_{1} \in A_{1}} \sum_{\substack{p_{2} \in A_{2} \\ p_{1} p_{2}^{a}-x y=1}} \chi\left(\frac{1}{2}\left(p_{1} p_{2}^{a}\right)^{1 / c}\right) .
$$

Так же, как это было при доказательстве теоремы 1, воспользуемся леммой 5 и выберем параметры $r, \Delta, \alpha, \beta$ двумя способами.

В данном случае сначала определим эти параметры так:

$$
r=[\ln n], \quad \Delta=\frac{1}{\ln ^{2} n}, \quad \alpha=\Delta, \quad \beta=\frac{1}{2}-\Delta .
$$

Обозначим через $\chi_{1}(x)$ функцию, существование которой следует из леммы 5. Затем при тех же $r$ и $\Delta$ положим $\alpha=-\Delta, \beta=1 / 2+\Delta$, а соответствующую функцию обозначим $\chi_{2}(x)$. Тогда из леммы 5 вытекает, что $\chi_{1}(x) \leqslant \chi(x) \leqslant \chi_{2}(x)$ и, следовательно,

$$
G_{11}(n) \leqslant G_{1}(n) \leqslant G_{12}(n),
$$

где

$$
G_{1 i}(n)=\sum_{p_{1} \in A_{1}} \sum_{p_{2} \in A_{2}} \sum_{\substack{x \leqslant \sqrt{n} \\ p_{1} p_{2}^{a}-x y=1}} \chi_{i}\left(\frac{1}{2}\left(p_{1} p_{2}^{a}\right)^{1 / c}\right), \quad i=1,2 .
$$

Если будут получены асимптотические формулы для $G_{11}(n)$ и $G_{12}(n)$ с совпадающими главными и остаточными членами, то из неравенства следует, что такая же формула будет верна и для $G_{1}(n)$.

3. Проведем подготовку к выводу асимптотической формулы для $G_{11}(n)$. Раскладывая функцию $\chi_{1}\left(\frac{1}{2}\left(p_{1} p_{2}^{a}\right)^{1 / c}\right)$ в ряд Фурье, получим

$$
G_{11}(n)=\left(\frac{1}{2}-2 \Delta\right) G(n)+R_{1}(n)+R_{2}(n),
$$

где

$$
\begin{aligned}
& R_{1}(n)=\sum_{0<|m| \leqslant \Delta^{-1} \ln n}\left|g_{m}\right|\left|V_{m}(n)\right|, \quad R_{2}(n)=\sum_{|m|>\Delta^{-1} \ln n}\left|g_{m}\right|\left|V_{m}(n)\right|, \\
& V_{m}(n)=\sum_{p_{1} \in A_{1}} \sum_{\substack{p_{2} \in A_{2} \\
p_{1} p_{2}^{a}-x y=1}} t_{2}\left(p_{1} p_{2}^{a}-1\right) e^{\pi i m\left(p_{1} p_{2}^{a}\right)^{1 / c}}, \quad t_{2}(k)=\sum_{\substack{x y=k \\
x \leqslant \sqrt{n} Q^{-1}}} 1,
\end{aligned}
$$

$g_{m}$ - коэффициент Фурье с номером $m$ для функции $\chi_{1}$.

Оценим $R_{2}(n)$. Из леммы 5 следует, что

$$
\left|g_{m}\right| \leqslant \frac{1}{\pi|m|}\left(\frac{r}{\pi \Delta|m|}\right)^{r} .
$$

Кроме того, $\left|V_{m}(n)\right| \leqslant G_{1}(n) \leqslant n$. Поэтому

$$
R_{2}(n)=O\left(n \sum_{|m|>\Delta^{-1} \ln n}\left(\frac{r}{\Delta}\right)^{r} m^{-r-1}\right)=O(1) .
$$

Оценим $R_{1}(n)$. Имеем

$$
\left|R_{1}(n)\right| \leqslant \sum_{0<|m|<\Delta^{-1} \ln n} \frac{1}{\pi|m|}\left|V_{m}(n)\right|, \quad\left|V_{m}(n)\right| \leqslant \sum_{n_{1} \leqslant \frac{n}{Q}}\left|\sum_{p_{2} \in A_{2}} t_{2}\left(n_{1} p_{2}^{a}-1\right) e^{\pi i m\left(n_{1} p_{2}^{a}\right)^{1 / c}}\right|,
$$


где $n_{1}$ пробегает множество натуральных чисел. Применяя неравенство Коши, получим

$$
\begin{aligned}
&\left|V_{m}(n)\right|^{2} \leqslant \frac{n}{Q} \sum_{n_{1} \leqslant n / Q}\left|\sum_{p_{2} \in A_{2}} t_{2}\left(n_{1} p_{2}^{a}-1\right) e^{\pi i m\left(n_{1} p_{2}^{a}\right)^{1 / c}}\right|^{2}= \\
&= \frac{n}{Q} \sum_{p_{2} \in A_{2}} \sum_{p_{2}^{\prime} \in A_{2}} \sum_{n_{1} \leqslant n / Q} t_{2}\left(n_{1} p_{2}^{a}-1\right) t_{2}\left(n_{1}\left(p_{2}^{\prime}\right)^{a}-1\right) e^{\pi i m\left(p_{2}^{a / c}-\left(p_{2}^{\prime}\right)^{a / c}\right) n_{1}^{1 / c}}= \\
&=\frac{n}{Q}\left(V_{0}(n)+V_{1}(n)\right),
\end{aligned}
$$

где сумма $V_{0}(n)$ соответствует слагаемым, в которых $p_{2}=p_{2}^{\prime}$, а в сумме $V_{1}(n) p_{2} \neq p_{2}^{\prime}$.

Оценим $V_{0}(n)$. Заметим, что из мультипликативности функции $\tau(n)$ и формулы для вычисления ее значений следует, что $\tau(a b) \leqslant \tau(a) \tau(b)$. Из этого замечания и леммы 6 следует, что

$$
V_{0}(n) \leqslant \sum_{p_{2} \in A_{2}} \sum_{n_{1} \leqslant \frac{n}{Q}} \tau^{2}\left(n_{1} p_{2}^{a}-1\right) \leqslant(a+1)^{2} Q^{1 / a} \sum_{n_{1} \leqslant n Q^{-1}} \tau^{2}\left(n_{1}\right) \ll n Q^{1 / a-1} \ln ^{3} n .
$$

Оценивая $V_{1}(n)$, заметим, что

где

$$
V_{1}(n) \leqslant \sum_{p_{2} \in A_{2}} \sum_{p_{2}^{\prime} \in A_{2}} f(n)
$$

$$
f(n)=\sum_{x_{1} \leqslant \sqrt{n} Q^{-1}} \sum_{x_{2} \leqslant \sqrt{n} Q^{-1}} s(m)
$$

и

$$
s(m)=\sum_{\substack{n_{1} \leqslant n Q^{-1} \\ n_{1} p_{2}^{a} \equiv 1 \\ n_{1}\left(p_{2}^{\prime}\right)^{a} \equiv 1\left(\bmod x_{1}\right)}} e^{2 \pi i \frac{m}{2}\left(p_{2}^{a / c}-\left(p_{2}^{\prime}\right)^{a / c}\right) n_{1}^{1 / c}}
$$

Будем считать без ограничения общности, что $\operatorname{gcd}\left(p_{2}, x_{1}\right)=1$ и $\operatorname{gcd}\left(p_{2}^{\prime}, x_{2}\right)=1$, так как в противном случае сумма будет равной нулю.

Пусть $p_{2} q_{2} \equiv 1\left(\bmod x_{1}\right)$ и $p_{2}^{\prime} q_{2}^{\prime} \equiv 1\left(\bmod x_{2}\right)$. Решим систему сравнений

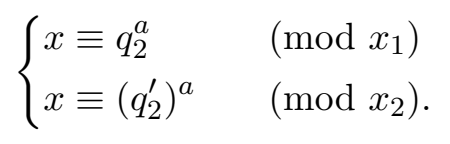

Она разрешима тогда и только тогда, когда $\operatorname{gcd}\left(x_{1}, x_{2}\right) \mid\left(q_{2}^{a}-\left(q_{2}^{\prime}\right)^{a}\right)$ и, в случае разрешимости, ее решение имеет вид $x=z_{2}+m D$, где $D=\left[x_{1}, x_{2}\right]$. Таким образом,

$$
s(m)=\sum_{\xi+l \leqslant n /(D Q)} e^{2 \pi i \varkappa(\xi+l)^{1 / c}}
$$

где

$$
\varkappa=\frac{m}{2}\left(p_{2}^{a / c}-\left(p_{2}^{\prime}\right)^{a / c}\right), \quad \xi=\frac{z_{2}}{D} .
$$

Очевидно, что $0<\xi<1$.

4. Для вывода асимптотической формулы для $G_{11}$ требуется оценить $s(m)$. Представим эту сумму в виде $s(m)=s_{1}(m)+s_{2}(m)$, где

$$
s_{1}(m)=\sum_{\frac{n}{D Q^{3 / 2}}<\xi+l \leqslant \frac{n}{D Q}} e^{2 \pi i \varkappa(\xi+l)^{1 / c}}, \quad s_{2}(m)=\sum_{\xi+l \leqslant \frac{n}{D Q^{3 / 2}}} e^{2 \pi i \varkappa(\xi+l)^{1 / c}} .
$$

Очевидно,

$$
\left|s_{2}(m)\right| \leqslant \sum_{\xi+l \leqslant \frac{n}{D Q^{3 / 2}}} 1 \leqslant \frac{n}{D Q^{3 / 2}}
$$


Поэтому

$$
s(m)=s_{1}(m)+O\left(\frac{n}{D Q^{3 / 2}}\right) .
$$

Разобьем сумму $s_{1}(m)$ на $O(\ln n)$ сумм вида

где

$$
\overline{s_{1}}(M)=\sum_{M<\xi+l \leqslant M_{1}} e^{2 \pi i \varkappa(\xi+l)^{1 / c}},
$$

$$
\frac{n}{D Q^{3 / 2}} \leqslant M<M_{1} \leqslant 2 M, \quad M_{1} \leqslant \frac{n}{D Q} .
$$

Поскольку $x_{1} \leqslant \sqrt{n} Q^{-1}$ и $x_{2} \leqslant \sqrt{n} Q^{-1}$, имеем $D=\left[x_{1}, x_{2}\right] \leqslant x_{1} x_{2} \leqslant n Q^{-2}$. Поэтому

$$
M \geqslant \frac{n}{D Q^{3 / 2}} \geqslant Q^{1 / 2}
$$

и, следовательно, сумма $\overline{s_{1}}(M)$ содержит «сравнительно много» слагаемых.

Будем оценивать $\overline{s_{1}}(M)$, считая, что $\frac{n}{D Q^{3 / 2}} \leqslant M \leqslant \frac{n}{D Q}$. Сначала рассмотрим случай, когда

$$
1 \leqslant D \leqslant n^{0,99} \Longleftrightarrow M \geqslant \frac{n^{0,01}}{Q^{1,5}}
$$

Если

$$
\frac{|\varkappa| M^{1 / c}}{M} \leqslant \frac{1}{10}
$$

то, приближая сумму интегралом, получим:

$$
\overline{s_{1}}(M)=\int_{M}^{M_{1}} e^{2 \pi i \varkappa(\xi+l)^{1 / c}} d l=O(1)=O\left(M^{1-1 / c}\right)
$$

В дальнейшем считаем, что $|\varkappa| M^{1 / c} / M>1 / 10$, и применим для оценки суммы $\overline{s_{1}}(M)$ метод ван дер Корпута. Определим натуральное число $k$ из условия

Если $k=2$, то

$$
\frac{1}{M^{2}}<\frac{|\varkappa| M^{1 / c}}{M^{k}} \leqslant \frac{1}{M}
$$

$$
\frac{|\varkappa| M^{1 / c}}{M^{2}} \asymp \frac{1}{M}
$$

Оценивая $\overline{s_{1}}(M)$ по второй производной, получаем, $\overline{s_{1}}(M)=O(\sqrt{M})$ при $k=2$.

Рассмотрим случай $k \geqslant 3$ и оценим $\overline{s_{1}}(M)$ по производной порядка $k$ (см. [10, с. 66-70]). Имеем $\overline{s_{1}}(M) \ll M^{1-\delta}$, где $\delta=\delta(k)>0$. Случай $D \leqslant n^{0,99}$ полностью рассмотрен.

Пусть теперь

$$
D>n^{0,99}, \quad \frac{|\varkappa| M^{1 / c}}{M}>\frac{1}{10} .
$$

Оценку $\overline{s_{1}}(M)$ будем вести по схеме оценки дзетовой суммы, принадлежащей И. М. Виноградову (см. $[10$, с. $66-70])$. Пусть $a=\left[M^{5 / 11}\right]$; тогда

$$
\left|\overline{s_{1}}(M)\right| \leqslant \frac{1}{a^{2}} \sum_{M<m \leqslant M_{1}}|W(m)|+2 a^{2},
$$

где

$$
W(m)=\sum_{u=1}^{a} \sum_{v=1}^{a} e^{2 \pi i \varkappa(\xi+m+u v)^{1 / c}} .
$$

Применим формулу Тейлора:

$$
(\xi+m+u v)^{1 / c}=\sum_{j=0}^{r}\left(\begin{array}{c}
1 / c \\
j
\end{array}\right)(\xi+m)^{1 / c-j}(u v)^{j}+\theta_{2}\left(\begin{array}{c}
1 / c \\
r+1
\end{array}\right)(\xi+m)^{1 / c-r-1} a^{2(r+1)}, \quad\left|\theta_{2}\right| \leqslant 1 .
$$


Таким образом,

$$
e^{2 \pi i \varkappa(\xi+m+u v)^{1 / c}}=e^{2 \pi i F(u v)}+2 \pi \theta_{3}|\varkappa|(\xi+m)^{1 / c}\left(\frac{a^{2}}{\xi+m}\right)^{r+1}, \quad\left|\theta_{3}\right| \leqslant 1,
$$

где

$$
F(u v)=\sum_{j=0}^{r}\left(\begin{array}{c}
1 / c \\
j
\end{array}\right)(\xi+m)^{1 / c-j}(u v)^{j} .
$$

Введем следующие обозначения:

Тогда

$$
x_{j}=\left(\begin{array}{c}
1 / c \\
j
\end{array}\right), \quad T=|\varkappa|(\xi+m)^{1 / c}, \quad \alpha_{j}=\frac{T \operatorname{sgn} \varkappa}{x_{j}\left(\xi+m_{j}\right)} .
$$

$$
\begin{gathered}
W(m)=W_{1}+2 \pi \theta_{4} T\left(\frac{a^{2}}{M}\right)^{r} a^{2} M^{-\frac{1}{11}}, \quad\left|\theta_{4}\right| \leqslant 1 \\
W_{1}=\sum_{u=1}^{a} \sum_{v=1}^{a} e^{2 \pi i F(u v)}=\sum_{u=1}^{a} \sum_{v=1}^{a} e^{2 \pi i\left(\alpha_{1} u v+\alpha_{2} u^{2} v^{2}+\cdots+\alpha_{r} u^{r} v^{r}\right)} .
\end{gathered}
$$

Выберем натуральное число $r$ из условия

$$
r-1<\frac{11 \ln T}{\ln M} \leqslant r
$$

и заметим, что, поскольку $D>0,99$, имеем $|\varkappa| \leqslant n^{0,99 / c}$. Поэтому из неравенства $M D \leqslant n / Q$ заключаем, что $M<n^{0,01}$. Следовательно,

$$
\frac{\ln T}{\ln M}>\frac{\ln \frac{1}{2} n^{0,99 / c}}{\ln n^{0,01}}>\frac{99}{c}-1 \geqslant \frac{97}{2},
$$

и указанный выбор $r$ возможен. Следуя схеме Виноградова, получим:

$$
\left|W_{1}\right| \leqslant c_{2} a^{2} e^{-\gamma_{1} \sqrt{\ln n}}, \quad c_{2}>0, \quad \gamma_{1}>0
$$

Следовательно,

$$
\bar{s}_{1}(M) \ll \frac{n}{Q D} e^{-\gamma \sqrt{\ln n}}, \quad s_{1}(m) \ll \frac{n \ln n}{Q D} e^{-\gamma \sqrt{\ln n}} .
$$

Используя полученные оценки, из (32)) находим

$$
s(m) \ll n Q^{-1} e^{-\gamma \sqrt{\ln n}} D^{-1} \ln n+n Q^{-1,5} D^{-1} \ll n Q^{-1} e^{-\gamma \sqrt{\ln n} / 2} D^{-1},
$$

где $D=\left[x, x_{2}\right]$.

5. Продолжим вывод асимптотической формулы для $G_{11}(n)$. Из формул $(26)$ и $(27)$ следует

$$
G_{11}(n)=\left(\frac{1}{2}-2 \Delta\right) G_{1}(n)+O\left(\sum_{0<|m|<\Delta^{-1} \ln n} \frac{1}{\pi|m|}\left|V_{m}(n)\right|\right),
$$

где $\Delta=1 / \ln ^{2} n$. Из (28) и (29) получаем

$$
\left|V_{m}(n)\right|^{2} \leqslant \frac{n}{Q} V_{1}(n)+O\left(n^{2} Q^{1 / a-2} \ln ^{3} n\right) .
$$

Поэтому для получения асимптотической формулы нужна оценка $V_{1}(n)$. Из $(30)$ имеем

$$
V_{1}(n) \ll \sum_{p_{2} \in A_{2}} \sum_{p_{2}^{\prime} \in A_{2}} f(n)
$$

где

$$
f(n)=\sum_{x_{1} \leqslant \sqrt{n} Q^{-1}} \sum_{x_{2} \leqslant \sqrt{n} Q^{-1}} s(m) .
$$


Подставив в эту формулу оценку (33), получим:

$$
f(n) \ll \frac{n}{Q} \exp \left(-\frac{\gamma}{2} \sqrt{\ln n}\right) \sum_{x_{1} \leqslant \sqrt{n} Q^{-1}} \sum_{x_{2} \leqslant \sqrt{n} Q^{-1}} D^{-1} .
$$

Оценим сумму в этом неравенстве:

$$
\begin{aligned}
\sum_{x_{1} \leqslant \sqrt{n} Q^{-1}} \sum_{x_{2} \leqslant \sqrt{n} Q^{-1}} D^{-1}= & \sum_{x_{1} \leqslant \sqrt{n} Q^{-1}} \sum_{x_{2} \leqslant \sqrt{n} Q^{-1}} \frac{\operatorname{gcd}\left(x_{1}, x_{2}\right)}{x_{1} x_{2}}= \\
= & \sum_{x_{1} \leqslant \sqrt{n} Q^{-1}} \sum_{x_{2} \leqslant \sqrt{n} Q^{-1}} \frac{1}{x_{1} x_{2}} \sum_{d \mid\left(x_{1}, x_{2}\right)} \varphi(d) \leqslant \\
& \leqslant \sum_{d \leqslant \sqrt{n} P^{-1}} \frac{\varphi(d)}{d^{2}}\left(\sum_{x \leqslant \sqrt{n} Q^{-1} d^{-1}} \frac{1}{x}\right)^{2} \ll \ln ^{3} n .
\end{aligned}
$$

Поэтому $f(n) \ll n \ln ^{3} n / Q^{2}$. Отсюда и из (30) следует, что

$$
\left|V_{1}(n)\right| \ll n^{2} Q^{1 / a-3} \ln ^{3} n .
$$

Используя оценки $(29)$ и (34), из (28) получим

то есть

$$
\left|V_{m}(n)\right|^{2} \ll n^{2} Q^{-2+\frac{2}{a}} e^{-\frac{\gamma}{4} \sqrt{\ln n}}+n^{2} Q^{-2+\frac{1}{a}} \ln ^{3} n \ll\left(\frac{n}{Q}\right)^{2} Q^{-2+2 / a} e^{-\gamma \sqrt{\ln n} / 4},
$$

Поэтому

$$
\left|V_{m}(n)\right| \ll n Q^{-1+1 / a} e^{-\gamma \sqrt{\ln n} / 8} .
$$

$$
\left|R_{1}(n)\right| \ll n Q^{-1+1 / a} e^{-\gamma \sqrt{\ln n} / 8} \ln ^{2} n .
$$

Подставляя оценки $(27)$ и (35) в (26), получаем асимптотическую формулу

$$
G_{11}(n)=\frac{1}{2} G(n)\left(1+O\left(Q^{-\eta}\right)\right), \quad \eta>0 .
$$

Аналогичными рассуждениями для $G_{12}(n)$ выводим асимптотическую формулу с такими же главным членом и остатком. Поэтому из $(25)$ следует, что для $G_{1}(n)$ верна асимптотическая формула

$$
G_{1}(n)=\frac{1}{2} G(n)\left(1+O\left(Q^{-\eta}\right)\right), \quad \eta>0 .
$$

Теорема 2 доказана.

\section{СПИСОК ЛИТЕРАТУРЫ}

1. Виноградов А. И. О плотностной гипотезе для $L$-рядов Дирихле// Изв. АН СССР. Сер. мат. -1965. - 29, № 4. - С. 903-934.

2. Виноградов И. М. Некоторое общее свойство распределения простых чисел// Мат. сб. - 1940. - 7. C. $365-372$.

3. Виноградов И. М. Метод тригонометрических сумм в теории чисел. - М.: Наука, 1971.

4. Виноградов И. М. Основы теории чисел. - СПб.-М.: Лань, 2004.

5. Гриценко С. А. Об одной задаче И. М. Виноградова// Мат. заметки. - 1986. - 39, № 5. - С. 625-640.

6. Гриценко C. А. Тернарная проблема Гольдбаха и проблема Гольдбаха-Варинга с простыми числами, лежащими в промежутках специального вида// Усп. мат. наук. - 1988. - 43, № 4 (262). - С. 203-204.

7. Гриценко С. А. Три аддитивные задачи// Изв. РАН. Сер. мат. - 1992. - 56, № 6 (9262). - С. 1198-1216.

8. Гриценко C. А., Зинченко Н. А. Об оценке одной тригонометрической суммы по простым числам// Науч. вед. БелГУ. Сер. мат. физ. - 2013. - 5 (148), № 30. - С. 48-52.

9. Зинченко H. А. Бинарная аддитивная задача с полупростыми числами специального вида// Чебышев. сб. - 2005. - VI, № 2 (14). - С. 145-162.

10. Карачуба A. А. Основы аналитической теории чисел. - М.: Наука, 1983.

11. Линник Ю. В. Дисперсионный метод в бинарных аддитивных задачах. - Л.: Изд-во ЛГУ, 1961. 
12. Хооли K. Применение методов решета в теории чисел. - М.: Наука, 1987.

13. Чанга M. E. Простые числа в специальных промежутках и аддитивные задачи с такими числами// Мат. заметки. - 2003. - 73, № 3. - C. 423-436.

14. Bombieri E. On the large sieve// Mathematica. — 1965. - 12. - P. 201-225.

15. Balog A., Friedlander K. J. A hybrid of theorems of Vinogradov and Piatetski-Shapiro// Pac. J. Math. 1992. - 156. - P. 45-62.

16. Titchmarsh E. C. A divisor problem// Rend. Circ. Mat. Palermo. - 1930. — 54. — P. 414-429.

17. Tolev D. I. On a theorem of Bombieri-Vinogradov type for prime numbers from a thin set// Acta Arithm. - 1997. - 81, № 1. - P. 57-68.

Зинченко Наталья Алексеевна

Белгородский государственный национальный исследовательский университет

E-mail: zinchenko@bsu.edu.ru 